\title{
FROZEN SHOULDER OR ADHESIVE CAPSULITIS
}

*Mr.Velmurugan

\begin{abstract}
:
Frozen shoulder is a poorly understood condition that typically involve substantial pain, movement restrictions and considerable morbidity. Although function improve overtime, full and pain free ranges, may not be restored in everyone. Frozen shoulder is also known as adhesive cpausulits . the treatment of this conditions, manipulation under anaesthesia has been associated with joint damage and may be no more effective than physiotherapy. Capsular release is another surgical procedure that is supported by expert opinion and published case series, but currently high quality research is not available.
\end{abstract}

Keywords: Frozen shoulder, physiotherapy

\section{Introduction:}

The shoulder is a complex anatomic structure that allow movement in many planes. Physician and patient alike don't often think about the important of the shoulder joint until its functions becomes compromised. The term "frozen shoulder" has been loosely applied to condition when shoulder is working at less than its optimal range. Because the shoulder joint is so complex, it is important to determine the practice the precise cause for loss of shoulder mobility ${ }^{1}$

\section{Definition:}

Frozen shoulder is a conditions that leads to pain and stiffness of shoulder. It is also known as adhesive capsulitis or shoulder contracture (Arkkila PE, 1996) ${ }^{5}$

\section{Incidence:}

It is estimated that upto1 in 20 people in the UK may be affected by frozen shoulder are between the ages of 40 and 60 . The conditions is more common in women than men. ${ }^{1}$
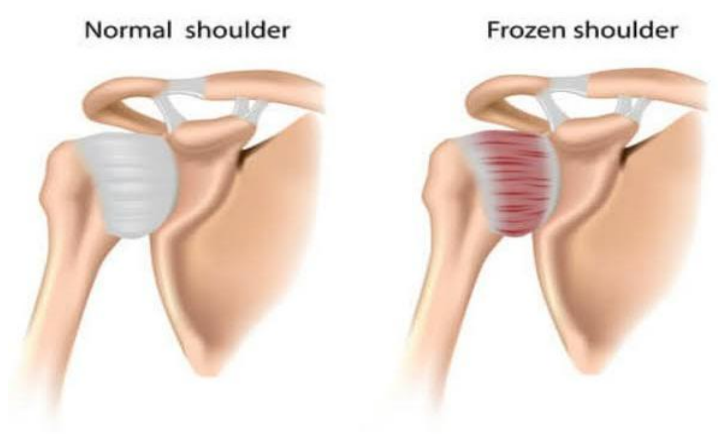

\section{Causes}

The causes of frozen shoulder are not fully understood. There is no clear connection to arm dominance or occupation. A few factors may put you more at risk for developing frozen shoulder 
Diabetes: (Affecting 10\%) to $20 \%$ of these individuals. The reason for is not known.)

$>$ Dupuytren's contracture: (A condition where small lumps of thickened tissue from in the hands and finger)

$>$ Immobilization.(Shoulder immobilized for a period of time due to surgery, a fracture, or other injury).

Other disease. Some additional medical problems associated with frozen shoulder include hypothyroidism, hyperthyroidism, Parkinson's disease, and cardiac disease.

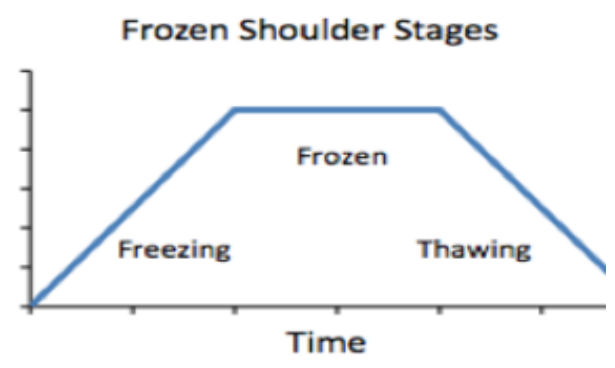

Stages:

In frozen shoulder, the shoulder capsule thickens and becomes tight. Stiff bands of tissue called adhesions. In many cases, there is less synovial fluid in the joint. The hallmark sign of this condition is being unable to move the shoulder either on own or with the help of someone else. It develops in three stages

\section{Freezing}

In the "freezing stage, the patient slowly has more and more pain. As the pain worsens, the shoulder loses range of motion. Freezing typically lasts from 6 weeks to 9 months.

\section{Frozen}

Painful symptoms may actually improve during this stage, but the stiffness remains. During the 4 to 6 months of the "frozen" stage, daily activities may be very difficult.

\section{Thawing}

Shoulder motion slowly improves during the "thawing stage. Complete return to normal or close to normal strength and motion typically takes from 6 months to 2 years.

\section{Clinical manifestation:}

$>$ Pain usually dull or aching. (Usually located outer shoulder area or upper arm).

Persistent stiffness in shoulder joint.

$>$ Difficult perform everyday taks such as

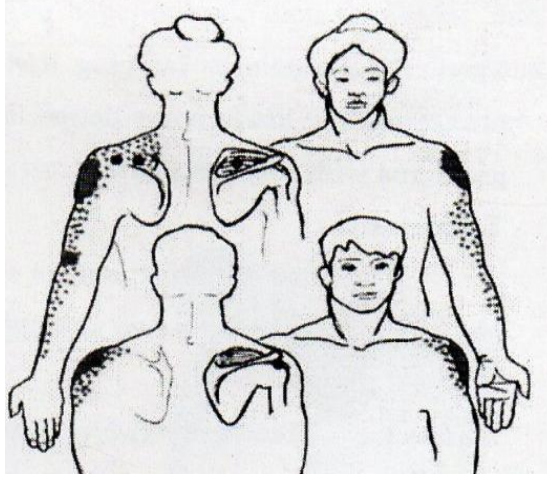


bathing. Dressing, driving and sleeping comfortably.

\section{Diagnostic evaluation:}

$>$ History collection (Age, sex and past medical and surgical history help in a part of diagnosis)

Physical examination: (Active and passive range of motion helps to know the extent of stiffness in shoulder)

$>$ X-ray (Helps to detect the dense structures of bone and joints.. It's to detect other problem such as arthritis)

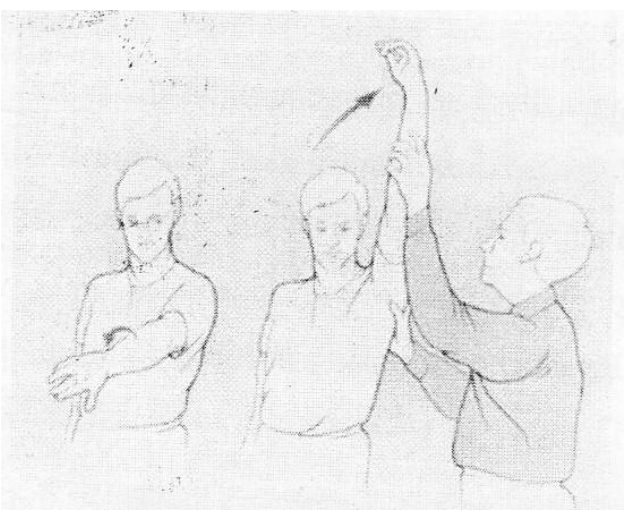

Magnetic resonance imaging (MRI) and ultrasound: (It can create better images of problems with soft tissues)

\section{Treatment:}

The focus of treatment is to control pain and restore motion and strength through physical therapy.

Nonsurgical Treatment: (More than $90 \%$ of patients improve with relatively simple treatments to control pain and restore motion.

Non-steroidal anti-inflammatory medicines.(Drugs like aspirin and ibuprofen reduce pain and swelling).

Steroid injections. (Cortisone is a powerful anti-inflmmatory medicine that is injection directly into shoulder joint).

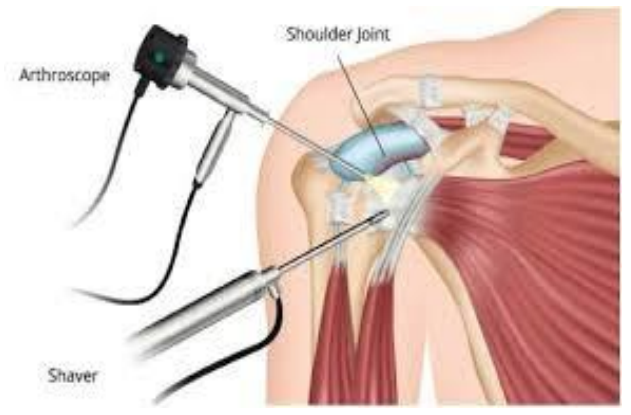

Physical therapy. Specific exercises will help restore motion. Therapy includes stretching or range of motion exercises for the shoulder. Sometimes heat is used to help loosen the shoulder up before the stretching exercises. (Example external rotation, Forward flexion, cross over arm stretch ect) Surgical treatment: (The goal of surgery for frozen shoulder is to stretch and release the stiffened joint capsule).

Manipulation under anesthesia. (During this procedure, the client is made to sleep. The which causes the capsule and scar tissue to stretch or 
tear. This releases the tightening and increases range of motion).

Shoulder arthroscopy. In this procedure, the surgeon will cut through tight portions of the joint capsule. This is done using pencil sized instruments inserted through small incisions around the shoulder).

\section{Nursing management:}

\section{Nursing assessment:}

$>$ Assessment of pain: (Verbal rating scale or numerical rating scale for pain level which ranging from mild to severe)

Assessment of Posture: (Cervical thoracic, Lumbar alignment and humeral and scapular position. Typical findings may include forward head position, protracted scapula, and excessive thoracic kyphosis)

Observation of affected extremity: (Observe client willingness to use affected upper extremities and alignment of bones \& soft tissues)

$>$ Palpation: (The nurse can palpate the scapular, cervical and shoulder girdle musculature for identification of tenderness)

\section{Goal:}

$>$ Relieving of pain

$>$ Increase the range of motion for all affected extremity

$>$ Promote comfort in affected extremity

Demonstration of normal postural alignment

Demonstrate high level of muscular performance on involved upper extremity

\section{Nursing interventions:}

$>$ Assess the client range of motion in affected extremity

$>$ Monitor vital signs

$>$ Assist in range of motion exercises

> Assist with physiotherapy for relief procedures (TENS-Trans Electrical Nerve Stimulation \& PENSPercutaneous Electrical Nerve Stimulation)

$>$ Provide Warm compress over shoulder physician order

$>$ Provide patient or family education

* Involve patient in rehabilitation programme

* Home exercise programme

* Pain management technique

Postural awareness education 
Demonstrate stretching exercise to reduce stiffness as per physician order

Pendulum exercise While leaning forward and holding onto a table or the back of a chair with your good arm, bend at the waist, allowing your affected arm back and forth like a pendulum, them in circles that start small and gradually grow larger as pain allows. Try this for about 2 or 3 minutes, several times a day.

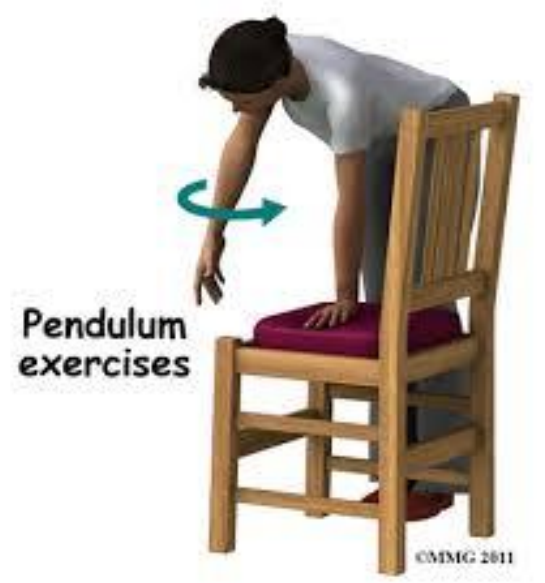

Wall climbing (to the side) Stand with your side to a wall so that your fingers can just touch it. Then turn so your body is turned slightly toward the will. Walk the fingers of your injured arm up the wall as high as pain permits. Hold that position for a count of 15 to 30 seconds. Walk your fingers down to the starting position.
Repeat 2 to 4 times, trying to reach higher each time.
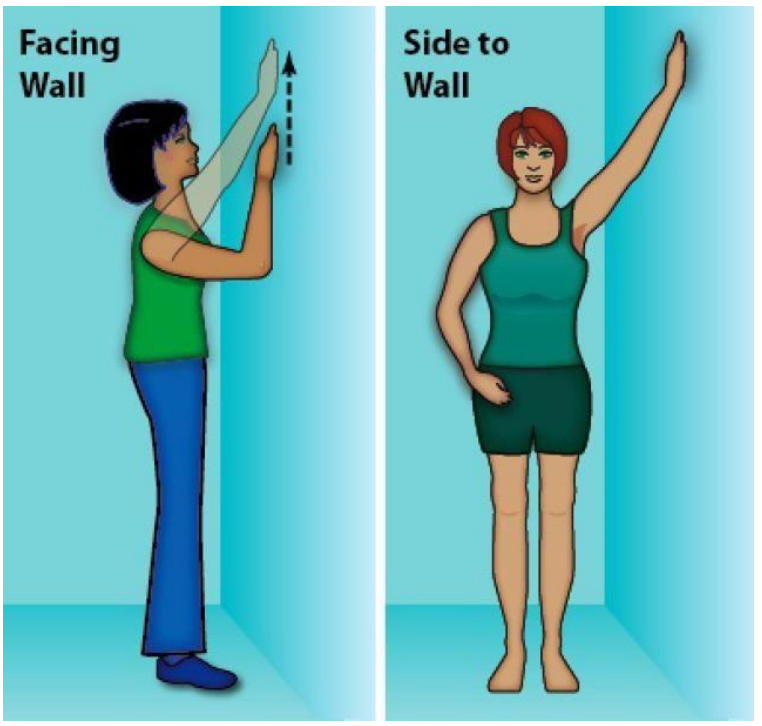

* Well climbing (to the front). Face a wall, standing so your fingers can just touch it. Walk the fingers of your affected arm up the wall as high as pain permits. Hold that position for a count of 15 to 30 seconds. Slowly walk your fingers to the starting position. Repeat 2 to 4 times, trying to reach higher each time.

\section{Reference:}

1. Neviaser JS. Adhesive capsulitis of the shoulder: a study of the pathologic findings in periarthritis of the shoulder. Journal of bone and joint surgery. 1945; 27:211-22.

2. Neviaser JS.Adhesive capsulitis of the shoulder. Medical Times. 1962;90: 783-807. 
3. Richards DB, Kibler WB. Sports related shoulder rehabilition. Journal of Musculoskeletal Medicine. 1997;14: 44-63.

4. Reeves B.The natural history of the frozen shoulder syndrome. Scand Journal of Rheumatology. 1975;4: 193-6.

5. Arkkila PE, Kantola IM, Viikari JS, Ronnemaa T.Shoulder capsulitis in types I and II diabetic patients. British medical journal. 1996;55: 907-14.

6. Karen baker MHS, Frozen shoulder care instruction. Health wish for every health decision. 2006-2015. 